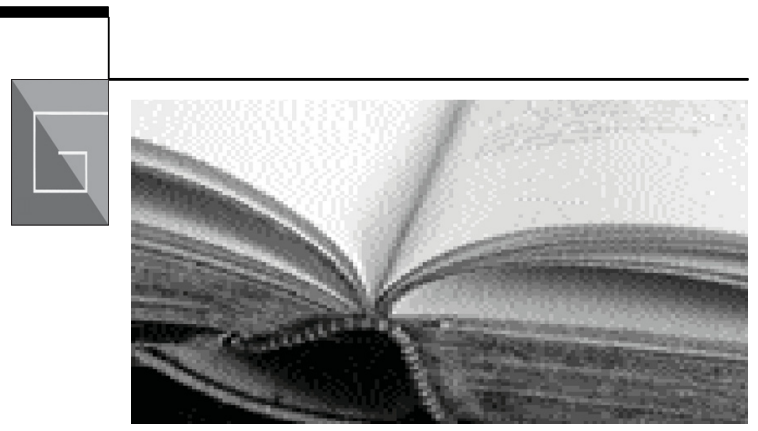

\title{
Les grands auteurs en finance
}

\author{
Michel Albouy et Gérard Charreaux (dir.) \\ Éditions Management \& Société (EMS), $2^{e}$ édition 2017, Caen, 711 p.
}

\section{$\mathrm{T}$}

oute publication académique a vocation à apporter une contribution, à des degrés divers et avec un impact plus ou moins grand, à l'édification du savoir, monument en perpétuelle évolution. La recension d'une publication, exercice classique s'il en est dans ce "Petit Monde » qu'est l'université, introduit un élément supplémentaire dans la relation entre un chercheur et son objet de recherche, via le regard du rédacteur de la recension sur cet objet de recherche, voire sur le chercheur luimême... Dans le cas d'une recension d'une publication portant sur un auteur connu et sur son œuvre (ex : ouvrage ou article biobibiographique), on franchit un échelon de plus dans la triangulation, le regard du recenseur portant tout à tour sur le rédacteur de la dite publication, sur l'auteur étudié et sur son œuvre, enfin, à travers eux sur le champ scientifique ainsi questionné. Que dire alors d'un ouvrage collectif qui rassemble un certain nombre de rédacteurs se partageant la tâche de présenter un certain nombre de "grands auteurs » : i.e. reconnus comme majeurs dans un champ disciplinaire donné. L'objectif de tels traités est de permettre au monde académique concerné - en premier lieu aux étudiants en cours de formation - de disposer d'un document pédagogique suffisamment vaste pour présenter le champ disciplinaire visé et suffisamment approfondi dans chacune de ses composantes pour permettre aux lecteurs d'avoir accès avec un minimum de fiabilité aux auteurs étudiés et à leurs œuvres respectives. Le regard du recenseur doit alors pouvoir se porter successivement sur les caractéristiques générales de l'ouvrage sous revue, sur son/ses coordinateur(s) et le collectif de contributeurs, sur les auteurs étudiés et les présentations successives de leurs travaux, enfin, sur la portée de cet ouvrage et son positionnement dans l'évolution du champ scientifique 
auquel il se rattache. C'est ce que nous allons tenter de faire.

\section{Aspects généraux}

Les Éditions Management \& Société (EMS), créées en Normandie en 1997, sont devenues, en une vingtaine d'années, d'une petite maison provinciale liée à une imprimerie, un des acteurs majeurs de l'édition universitaire dans son secteur de référence : gestion-management et actualité économique; succès indéniable porté par la croissance de ce secteur éducatif dont les effectifs sont devenus les plus fournis de l'enseignement supérieur français.

Au sein de cette nouvelle maison d'édition, la collection « Grands auteurs » a été créée dans le but de «présenter de façon synthétique les apports des auteurs majeurs des disciplines» du champ éditorial concerné. Le principe suivi a été le suivant : les responsables de la collection - Gérard Charreaux, Patrick Joffre et Gérard Koenig, tous les trois universitaires reconnus dans le champ des sciences de gestion - esquissent un projet éditorial concernant telle ou telle thématique et en confient la réalisation à un (ou plus) collègue(s) spécialiste(s) de la dite thématique. $\mathrm{Ce}$ (s) responsable(s) à leur tout, finalise(nt) le projet éditorial, notamment en termes de choix des « grands auteurs » à présenter et des collègues français/francophones connaissant bien ces auteurs et leurs travaux. À ce jour, une dizaine d'ouvrages collectifs sur les « grands auteurs » ont ainsi été publiés, couvrant les grandes thématiques du champ disciplinaire ; certains en étant à la troisième édition.

La finance faisait évidemment partie de ces thématiques et un premier ouvrage sur « Les grands auteurs en finance » a été publié en
2003, sous la direction de Michel Albouy. Il présentait, en une douzaine de chapitres, les grands noms de la finance académique internationale - en l'occurrence américaine - dont plusieurs avaient été honorés du Prix de la banque de Suède, dit «Nobel d'économie ». Comme le principe de la collection le posait, chacun de ces grands auteurs était présenté par un (parfois deux) collègues francophones, eux-mêmes universitaires connus sur la scène française de la recherche en finance (notamment via l'association de référence que constitue l'AFFI).

L'ouvrage sous revue est présenté comme une deuxième édition du précédent. Son examen fait apparaître qu'il va bien au-delà des habituelles rééditions qui se bornent souvent à des mises à jour avec, ici ou là, quelques ajustements. C'est en fait un nouveau volume qui nous est proposé : ceci sous divers aspects majeurs :

- au niveau de la coordination de l'ensemble de l'ouvrage, on est passé de un à deux, le coordinateur initial, Michel Albouy, faisant équipe avec Gérard Charreaux, déjà contributeur dans la précédente édition (chapitre concernant Jensen) et par ailleurs - comme on l'a rappelé - un des responsables de cette collection «grands auteurs » créée par EMS. La complémentarité de ces deux collègues était un atout en faveur d'un élargissement du champ financier tant vers les domaines généraux du management et de la stratégie que de celui de la gouvernance.

- au niveau des contributeurs, ces derniers sont passés de treize pour la première édition à trente-deux pour la seconde, mobilisant non seulement les chefs de file de la discipline financière en France, mais nombre de leurs collègues plus jeunes et de 
ce fait moins connus, assurant, à cet égard, une heureuse relève générationnelle.

- au niveau des « grands auteurs » étudiés, leur nombre est passé de onze à vingt-deux (cf tableau 1), doublement quantitatif qui s'accompagne - comme on le verra ci-après - d'une évolution significative en termes de positionnement et d'orientation théorique. - in fine, cette seconde édition de l'ouvrage sur « Les grands auteurs en finance », parue en août 2017, correspond à un impressionnant volume de plus de 700 pages, à comparer aux 310 pages de la première édition.

\section{Structure et contenu de l'ouvrage}

L'ouvrage commence par une introduction, signée par les deux coordinateurs et intitulée : « La construction de la théorie financière moderne : de la finance néoclassique à la finance néoinstitutionnelle et comportementale. Une introduction aux grands auteurs en finance ». En une cinquantaine de pages, Michel Albouy et Gérard Charreaux expliquent leur projet éditorial pour cette nouvelle édition; citons-les : «Nous allons, dans un premier lieu, préciser les contraintes qui nous ont orientés pour établir la liste des grands auteurs et les modalités précises de la sélection qui a été faite. En deuxième lieu, nous présenterons la logique que nous avons adoptée pour structurer l'ordre de présentation des différentes contributions dont nous donnerons un bref aperçu. En troisième lieu, nous tenterons de tirer un certain nombre d'enseignements de ce voyage en pays de finance ». Je recommande à tout lecteur de lire cet important chapitre introductif avant de commencer la lecture de l'ouvrage, puis de le relire après... Nous y reviendrons à la fin de la présente recension.
L'ouvrage se décline ensuite en autant de chapitres qu'il y a de «grands auteurs » étudiés, chacun d'entre eux étant caractérisé par un titre qui est apparu aux rédacteurs comme le plus significatif de l'apport de ses travaux au monde de la recherche en finance. Ainsi, le lecteur est invité à voyager des « fondations de la théorie moderne du portefeuille » (chap. 1) avec H. Markowitz dont les travaux datent des débuts des années cinquante, jusqu'à « la prise en compte des interactions des acteurs sur le marché dans la finance comportementale » (chap. 22) avec J. C. Stein dont les analyses sont actuellement en plein développement. C'est donc plus d'un demi-siècle d'histoire de la recherche en finance que les différents contributeurs à cet ouvrage collectif nous incitent à parcourir.

$\mathrm{Au}$ plan formel, les différents chapitres se lisent bien - ce qui n'est pas toujours le cas en matière de recherche en finance - les rédacteurs ayant reçu vraisemblablement des consignes en ce sens, compte tenu de la vocation pédagogique de l'ouvrage. On notera quelques présentations particulières : ainsi celle de M. S. Scholes (chap. 7) s'appuie sur le discours de réception prononcé par B. Jacquillat lors d'une remise de doctorat honoris causa à l'intéressé (en 1989 à Paris-Dauphine) ; ou encore celle R. Roll (chap. 10) que les présentateurs - E. de Bodt et M. Levasseur - ont conçu sous la forme d'un récit de voyage... Notons également des disparités dans les notes biographiques des auteurs recensés - de quelques lignes à plusieurs pages - notes d'information qu'il conviendrait d'harmoniser a minima (par exemple en donnant l'année de naissance qui manque parfois...). $\mathrm{Au}$ plan de la structure de l'ouvrage, les coordinateurs ont choisi de «catégoriser » 
les différents auteurs étudiés, en distinguant plusieurs sous-ensembles :

$l^{\text {ère }}$ partie : « La constitution du paradigme dominant: la finance néoclassique » : regroupant les auteurs les plus emblématiques (Markovitz, Sharpe, Miller, Fama, Ross, Black, Scholes, Merton), tous déjà présents dans la première édition.

$2^{e}$ partie : «Des fissures importantes dans l'édifice néoclassique »: présentant des auteurs plus critiques (Stiglitz, Roll, Harvey, Titman).

$3^{e}$ partie : «Une première voie de reconstruction : la finance néoinstitutionnelle contractuelle »: avec plusieurs auteurs connus (Myers, Jensen, Stulz, Diamond, Hart, Zingales).

$4^{e}$ partie : «Vers un nouveau paradigme dominant : la finance comportementale » : présentée via plusieurs de ses promoteurs (Thaler, Shiller, Shleifer, Stein).

Comme on le voit, cette catégorisation qui a servi à structurer l'ouvrage n'est pas innocente ; elle exprime le projet éditorial suivi par les coordinateurs et annoncé dans le chapitre introductif : retracer, à travers la présentation des différents grands auteurs retenus, une brève histoire de la recherche académique en finance, du moins telle qu'ils la ressentent.

Cette impression est confortée lorsqu'on analyse, plus en détail, les caractéristiques des auteurs étudiés. On peut dresser un tableau récapitulatif présentant successivement, pour chacun d'entre eux, dans l'ordre d'apparition dans l'édition 2017 (et un rappel éventuel de cet ordre dans l'édition 2003), leur pays (parfois deux), les dates de naissance (et éventuellement de décès), la date et l'université d'obtention du doctorat, enfin quelques éléments de notoriété comme la date d'entrée dans le Top 20 des citations de Google Scholar (GS) ${ }^{1}$, la présidence de l'American Finance Association (AFA) et, évidemment, le Prix de la Banque de Suède dit « Nobel » d'économie (cf. tableau 1).

Le résultat est édifiant lorsqu'on examine les caractéristiques de ce tableau

1) Observons tout d'abord qu'il n'y a aucune femme parmi ces « grands auteurs en finance ». Ce n'est évidemment pas un choix sexiste des responsables de l'ouvrage, mais un constat de leur part d'une moindre notoriété des auteurs féminins dans le domaine étudié - la finance - qui reste un univers très masculin, tant au niveau des chercheurs que des praticiens. À méditer... 2) La répartition par âges montre une évolution entre les deux éditions : alors que la première concernait des auteurs nés entre 1923 (Miller) et 1940 (Myers), la nouvelle édition s'intéresse à des auteurs plus récents, avec notamment plusieurs « sixties» (Stein, Shleifer, Zingales). Si la période écoulée entre les deux éditions (2003-2017) explique logiquement cette évolution, cette dernière fait ressortir un souci d'ouverture sur des auteurs plus contemporains. In fine, sur les vingt-deux auteurs étudiés, la quasi-totalité est encore en vie, seuls trois d'entre eux étant décédés : le doyen d'âge Miller depuis 2000, Black prématurément en $1995^{2}$, enfin Ross cette année 2017.

3) Une autre évidence est la domination quasi exclusive des États-Unis d'Amérique.

1. Les coordinateurs se sont en effet appuyés, entre autres critères, sur les citations via Google Scholar (cf. p. 54-55).

2. Décès qui ne lui a pas permis de recevoir le « Nobel » attribué en 1997 aux travaux sur les options menées avec Scholes et Merton. 
Tableau 1 - Tableau récapitulatif des différents auteurs

\begin{tabular}{|c|c|c|c|c|c|c|c|c|}
\hline 2017 & 2003 & Nom & Pays & Dates & PhD Université & GS & AFA & Nobel \\
\hline \multicolumn{9}{|c|}{$1^{\text {ère }}$ partie } \\
\hline 1 & 1 & H. M. Markowitz & USA & $1927-$ & 1952 Chicago & 1952 & 1982 & 1990 \\
\hline 2 & 2 & W.F. Sharpe & USA & $1934-\ldots$ & 1961 UCLA & 1964 & 1980 & 1990 \\
\hline 3 & 3 & M. H. Miller & USA & $1923-2000$ & 1952 Hopkins & 1958 & 1976 & 1990 \\
\hline 4 & 4 & E. F. Fama & USA & $1939-\ldots$ & 1964 Chicago & 1980 & & 2013 \\
\hline 5 & 10 & S. A. Ross & USA & 1944-2017 & 1969 Harvard & & 1988 & \\
\hline 6 & 7 & F. S. Black & USA & 1938-1995 & 1964 Harvard & 1973 & 1985 & \\
\hline 7 & 6 & M. S. Scholes & USA & $1941-\ldots$ & 970 Chicago & 1973 & 1990 & 1997 \\
\hline 8 & 8 & R. C. Merton & USA & $1944-\ldots$ & 1970 MIT & 1973 & 1986 & 1997 \\
\hline \multicolumn{9}{|c|}{$2^{\mathrm{e}}$ partie } \\
\hline 9 & & J. E. Stiglitz & USA & $1953-\ldots$ & 1967 MIT & 1981 & & 2001 \\
\hline 10 & 11 & R. Roll & USA & $1939-\ldots$ & 1968 Chicago & & 1987 & \\
\hline 11 & & C. R. Harvey & $\mathrm{Ca} / \mathrm{USA}$ & $1958-\ldots$ & 1986 Chicago & & 2016 & \\
\hline 12 & & S. Titman & USA & $1954-\ldots$ & 1981 Carnegie & & 2012 & \\
\hline \multicolumn{9}{|c|}{$3^{\mathrm{e}}$ partie } \\
\hline 13 & 9 & S. C. Myers & USA & $1940-\ldots$ & 1966 Stanford & 1977 & 1983 & \\
\hline 14 & 5 & M. C. Jensen & USA & $1939-\ldots$ & 1968 Chicago & 1976 & 1992 & \\
\hline 15 & & R. M. Stulz & USA & $1944-\ldots$ & 1980 MIT & & 2004 & \\
\hline 16 & & D. W. Diamond & USA & $1953-\ldots$ & 1980 Yale & & 2003 & \\
\hline 17 & & O. Hart & GB/USA & $1948-\ldots$ & 1974 Princeton & & & 2016 \\
\hline 18 & & L. Zingales & It/USA & $1963-\ldots$ & 1992 MIT & & 2014 & \\
\hline \multicolumn{9}{|c|}{$4^{\mathrm{e}}$ partie } \\
\hline 19 & & R. Thaler & USA & $1945-\ldots$ & 1974 Rochester & & & 2017 \\
\hline 20 & & R. J. Shiller & USA & $1946-\ldots$ & 1972 MIT & & & 2013 \\
\hline 21 & & A. Shleifer & USA & $1961-\ldots$ & 1986 MIT & 1997 & & \\
\hline 22 & & J. C. Stein & USA & $1960-\ldots$ & 1986 MIT & & 2008 & \\
\hline
\end{tabular}

Note : GS : Google Scholar, AFA : American Finance Association. 
Les onze auteurs recensés dans la première édition étaient américains. Dans la seconde, on en note quelques-uns qui le sont pas apparemment : le canadien Harvey, l'anglais Hart, l'italien Zingales; mais un examen plus attentif de leur parcours fait apparaître une forte imprégnation étatsunienne, notamment par leurs formations doctorales effectuées dans des grandes universités de ce pays. Plus qu'une domination en termes de nationalité, la répartition des affiliations doctorales fait apparaître une domination sans partage du système universitaire en place aux États-Unis.

$\mathrm{Au}$ sein de ce système, on observe le rôle prépondérant des plus grands établissements ; avec, là également, une évolution relative : alors que dans la première édition, l'université de Chicago avait une position ultra dominante, avec cinq des onze auteurs issus de ses rangs (Markowitz, Fama, Jensen Scholes, Roll), justifiant son image de « Mecque de la finance mainstream », cette position prépondérante est contestée dans la nouvelle édition par un autre établissement prestigieux - le MIT à Boston - où sept des vingt-deux auteurs recensés ont effectué leur $\mathrm{PhD}$ (contre six seulement à Chicago). Les autres universités, bien que souvent ellesmêmes de grand renom (Harvard, Princeton, Stanford, UCLA, Yale..), ne semblent jouer qu'un rôle marginal, alors même qu'elles sont souvent très actives dans le domaine de la formation et de la recherche en finance.

4) Les critères de notoriété choisis par les coordinateurs - citations dans Google Scholar, présidence de l'AFA, obtention du Nobel - paraissent probants, puisque les 22 auteurs retenus cumulent onze présences dans le Top 20 de GS, 16 présidences d'AFA, 10 prix Nobel ; cette domination étant évidemment plus nette pour les plus anciens (notamment ceux de l'édition 2003) ; les autres peuvent cependant espérer suivre leur voie... ${ }^{3}$. On observera cependant que ces critères renforcent encore le poids du monde anglophone et plus particulièrement des États-Unis, ce qui entraîne un biais méthodologique, à nos yeux évident, au niveau des critères de sélection.

\section{Portée de l'ouvrage : une étape significative dans le renouveau du corpus en finance}

Au terme de cette recension, nous souhaiterions donner notre sentiment sur cet ouvrage ; plus précisément notre ressenti de lecteur empathique envers le projet éditorial et ses concepteurs, admiratif du résultat de leur travail collectif, mais qui souhaiterait les voir aller plus loin.

\section{Un projet éditorial courageux}

Le responsable de la première édition des « Grands auteurs de la finance » aurait pu se contenter d'une mise à jour a minima de son ouvrage de 2003, en demandant aux contributeurs d'actualiser les chapitres concernant les onze auteurs présentés, à la rigueur en y ajoutant un douzième comme R. J. Shiller qui était déjà très connu dans le milieu académique, avant l'attribution en 2013 du Nobel d'économie ${ }^{4}$. Il a eu le mérite de ne pas s'en

\footnotetext{
3. Ainsi un des auteurs retenus en 4 partie (R. Thaler - chap. 19) a obtenu le Nobel d'économie en octobre 2017, donc postérieurement à la sortie de l'ouvrage sous revue...

4. Prix attribué en même temps à E. Fama, pur produit de l'école de Chicago, alors même que les positions scientifiques de ces deux auteurs se situent à l'opposé l'un de l'autre ; réunion œcuménique qui a laissé pantois les représentants d'autres disciplines et qui n'a pas contribué à la crédibilité de la science économique.
} 
contenter et de se lancer, en tandem avec un des responsables de la collection, dans une rénovation d'envergure. À notre sens, ils ont eu raison de tenter de le faire car la nouvelle crise financière, née avec l'affaire des subprimes et les excès de la titrisation, rendait nécessaire une telle rénovation ${ }^{5}$.

\section{Une réalisation impressionnante mais forcément inachevée}

Au terme de la lecture attentive de ce gros volume et d'une relecture - comme suggérée supra - de son chapitre introductif, nous avons des sentiments mêlés :

- une réelle admiration pour le travail accompli : cette édition 2017 apporte une substantielle plus-value par rapport à celle de 2003, non seulement par le nombre d'auteurs présentés mais en situant ces auteurs et leurs travaux dans une perspective de renouvellement du corpus académique en finance. Dans un langage kuhnien, les coordinateurs parlent de « paradigme dominant », de «fissures importantes », de « voie de reconstruction», de « nouveau paradigme »... jusqu'où iront-ils ? Nous sentons leurs excommunions poindre.... - un constat d'un processus forcément inachevé. Les coordinateurs auraient pu aller plus loin dans cette relève de paradigme, ce qui les aurait amenés, d'une part, à ajouter à la liste des chercheurs comme Michel Aglietta, Gaël Giraud, Paul Jorion, Steve Keen, Donald A. MacKenzie, André Orléan, Nassim Nicholas Thaleb, Christian Walter... et, d'autre part, à considérer qu'à force d'être fissuré, le temple néoclassique construit à Chicago devait être remplacé et que, en termes de modélisation, des Benoit Mendelbrot ou René Thom pouvaient prendre la place qui avait été celle de Hary Markowicz et William Sharpe. C'eut été une relève complète de paradigme au sens kuhnien... Mais, avec sagesse, et s'agissant d'un manuel destiné principalement à un public d'étudiants, nos collègues ont préféré avancer step by step ; laissant à une future édition le soin de confirmer l'étape actuelle et d'en proposer une nouvelle.

Roland PEREZ

5. Cf. R. Pérez (2010) « Analyse de la crise financière ou crise de l'analyse financière ? », Management \& Avenir, $\mathrm{n}^{\mathrm{o}} 35$, mai, p. 170-194. 